\title{
BMJ Open Association of resilience with health- related quality of life and depression in multiple myeloma and its precursors: results of a German cross-sectional study
}

\author{
Imad Maatouk, ${ }^{1,2}$ Susanne He ${ }^{3}$ Natalia Becker, ${ }^{4}$ Manuela Hummel, ${ }^{4}$ \\ Stefan Hemmer, ${ }^{5}$ Michaela Hillengass, ${ }^{3}$ Hartmut Goldschmidt, ${ }^{3,6}$ \\ Mechthild Hartmann, ${ }^{1}$ Dieter Schellberg, ${ }^{1}$ Wolfgang Herzog, ${ }^{1}$ Jens Hillengass ${ }^{3}$
}

To cite: Maatouk I, He S, Becker N, et al. Association of resilience with health-related quality of life and depression in multiple myeloma and its precursors: results of a German cross-sectional study. BMJ Open 2018:8:e021376. doi:10.1136/ bmjopen-2017-021376

- Prepublication history and additional material for this paper are available online. To view these files, please visit the journal online (http://dx.doi. org/10.1136/bmjopen-2017021376).

Received 27 December 2017 Revised 13 June 2018 Accepted 29 June 2018

Check for updates

(c) Author(s) (or their employer(s)) 2018. Re-use permitted under CC BY-NC. No commercial re-use. See rights and permissions. Published by BMJ.

For numbered affiliations see end of article.

Correspondence to Dr Imad Maatouk; imad.maatouk@med.uniheidelberg.de

\section{ABSTRACT}

Objectives To investigate the relation between resilience, health-related quality of life (HRQOL) and depression in multiple myeloma (MM) and its premalignant stages. MM is one of the most frequent haematological disorders. It is regularly preceded by asymptomatic stages of the disease namely monoclonal gammopathy of undetermined significance (MGUS) and smouldering multiple myeloma (SMM). Survivors have to cope with mental and physical impairment in terms of HRQOL and depression. The concept of resilience refers to a person's ability to adapt to adversity. Design Cross-sectional study.

Setting MM outpatient department at a University Hospital in Germany (tertiary care).

Participants 292 consecutive patients from our MM outpatient department.

Outcome measures HRQOL, depression and psychological resilience were assessed with validated questionnaires. Results Regression analyses were performed to determine associations between resilience, $\mathrm{HRQOL}$ and depression. 98 patients (33.6\%) had a new diagnosis of active MM, 106 patients (36.3\%) were already treated for MM and 88 patients had the diagnosis of a precursor (MGUS or SMM; 30.1\%) of MM. Multivariate linear regression analyses revealed a strong positive impact of resilience on physical (b 7.20; $95 \% \mathrm{Cl} 4.43$ to $9.98 ; \mathrm{p}<0.001$ ) and mental (b $12.12 ; 95 \% \mathrm{Cl}$ 9.36 to $14.87 ; p<0.001)$ HRQOL. Ordered logistic regression analysis showed that the odds for higher depression severity were lowered for individuals with a high level of resilience in comparison to the individuals with a low level of resilience (OR $0.11 ; 95 \%$ Cl 0.06 to $0.19 ; p<0.001$ ).

Conclusions Resilience may be a protective factor in the disease trajectory of MM and its precursors. As a next step, future research should focus on longitudinal assessments at various time points to elucidate the role of resilience in one of the most frequent haematological malignancies.

\section{BACKGROUND}

Multiple myeloma (MM) is one of the most frequent haematological malignancies with increasing incidence and prevalence due to
Strengths and limitations of this study

- Our study is the first to investigate the impact of resilience in the course of disease of multiple myeloma.

- Another strength of the study is its large sample size of 292 patients.

- Health-related quality of life (HRQOL) and other psychosocial constructs were measured with validated questionnaires.

- The main limitation of our study is related to its cross-sectional design that does not allow for either temporal or causal inferences.

- For measurement of HRQOL, we used a generic questionnaire.

demographic change and improvements in therapy. It is regularly preceded by asymptomatic stages namely monoclonal gammopathy of undetermined significance (MGUS) and smouldering multiple myeloma (SMM) with a risk of progression of approximately $1 \%$ and $10 \%$ per year, respectively. ${ }^{12}$

As a result of improvements in therapeutic interventions such as high-dose chemotherapy with autologous stem cell transplantation (ASCT) and introduction of novel agents, survival after diagnosis of MM has been extended impressively even in elderly patients. ${ }^{3}$ Survivors of MM have to cope with numerous symptoms due to organ damage caused by the disease as well as with the psychosocial burden over years. Long-lasting effects due to the drugs toxicities may be apparent after its completion. ${ }^{4}$

Due to the by definition absence of symptoms in MGUS and SMM, respectively, most patients do not need treatment right away but have to be monitored in an adequate manner. 
However, in all phases of the disease trajectory (including premalignant stages) patients may experience impairment of physical and mental health-related quality of life (HRQOL) ${ }^{45}$ HRQOL 'refers to the physical, psychological and social domains of health, seen as distinct areas that are influenced by a person's experiences, beliefs, expectations and perceptions'. ${ }^{6}$ Clinical observations show that older patients more often tend to name physical symptoms/limitations than mental difficulties.

In premalignant stages of MM, the awareness of the risk of developing full-blown MM could impair the perception of one's own physical quality of life. From a clinical perspective, this is a burden for many patients. However, there is no single published study that investigated HRQOL or psychological burden in patients with MGUS and SMM.

Prior studies have shown that about $25 \%$ of patients with MM suffer from high mental strain and depressive symptoms. ${ }^{78}$ Since the psychosocial dimensions of HRQOL were found to be independent prognostic factors in patients with $\mathrm{MM},{ }^{9}$ it is important to gain more knowledge about protective factors, contributing to the patient's ability to cope with the situation. Complementary to the study of impairment, researchers postulate the study of positive constructs such as resilience that could be important to maintain HRQOL and mental health in the course of a disease.

The concept of resilience was developed concurrently in many disciplines and contexts. Initially, it was viewed as a trait limited to few individuals that succeeded in life despite adverse circumstances during their childhood. Later studies focused on a developmental perspective and an investigation of paediatric cancer survivors. ${ }^{10}$ The concept of resilience has been well studied in recent decades in the field of cancer. In a recently published comprehensive review of resilience in adult cancer care, the authors conclude to 'define resilience in adult patients with cancer and survivors as a dynamic process of facing adversity related to the cancer experience'. ${ }^{10}$ It is conceptualised as the ability to bounce back from highly adverse conditions like a serious health problem. Studies in patients with different cancer types (eg, breast, lung, gastric cancer) suggest positive associations between resilience and HRQOL ${ }^{11-14}$ and lower emotional distress, respectively. ${ }^{15} 16$ However, there is a paucity of research evaluating the possible relevance of resilience in patients with MM. Myeloma is still incurable today. In the preliminary stages (without treatment), the uncertainty represents a strong burden and after the possibly achieved remission (after treatment), there is a high risk of recurrence. ${ }^{4}$ Furthermore, men are predominantly affected at a median age of 70 years. This group is known to have less access to psycho-oncological interventions, often evaluated in younger patients with breast cancer. ${ }^{17}$ Therefore, the nature of protective factors such as resilience in MM and its precursors has to be elucidated.

The purpose of this study was therefore to examine associations between resilience, HRQOL and depressive symptoms in a large sample of adult patients with MM, SMM and MGUS.

The following research questions were addressed:

Is there a significant relation between resilience and mental and physical HRQOL, respectively? Is there a significant relation between resilience and depression severity?

Further, we wanted investigate possible differences in the associations between resilience and the various outcomes (HRQOL and depression, respectively) for the different stages of MM. To the best of our knowledge, this is the first study to investigate the impact of resilience in the course of disease of MM.

\section{PATIENTS AND METHODS}

\section{Design and study population}

Questionnaires to assess different levels of HRQOL are part of a screening measure at time of first presentation within the clinical routine at our outpatient department. All patients who made the first appointment from November 2014 to April 2016 received a letter before they met the doctor in the hospital. This letter included information on the survey and a psychosocial questionnaire package. During the aforementioned period, all patients who had a first presentation at the centre with a diagnosis of MM, SMM and MGUS who had sufficient skills in reading and writing German were eligible for study participation. Exclusion criteria were as follows: cognitive impairment (ie, not being able to follow the informed consent or other indications that the contents of the procedure cannot be followed) and serious psychiatric illnesses at the time of presentation. Severe psychiatric symptoms were defined as follows: requiring an immediate treatment such as acute suicidal tendencies, psychotic symptoms, dissociation or flash backs and severe addictive diseases. The questionnaires were to be completed at home and given to a staff member at the first appointment in the clinic. Participation was voluntary, and participants could withdraw at any time without any consequences.

\section{Measurements}

HRQOL was assessed with the short form health survey12 , a widely used generic questionnaire. ${ }^{18} 19$ HRQOL is measured by two summarised components: a Physical Component Score (PCS) and a Mental Component Score (MCS). A higher score in the respective summary scale indicates a higher quality of life.

The Patient Health Questionnaire-9 (PHQ-9) was used to assess the presence and severity of depression symptoms. ${ }^{20}{ }^{21}$ The proven psychometric properties of the PHQ have been reported in various large-scale studies. The total score of all items of the PHQ-9 ranges between 0 and 27 points. A range from 0 to 4 points suggests no clinically significant symptoms, 5-9 points indicate mild depressive symptoms, 10-14 points refer to moderate symptoms and 15-27 points suggest the existence of 
severe depressive symptoms. Resilience was measured by the short form of the Resilience scale-13 (RS-13), a well validated questionnaire derived from the original scale of Wagnild and Young. ${ }^{22} 23$ The total score of all items ranges between 13 and 91 points. Respective ranges were used to categorise the 'level of resilience'. A range from 13 to 66 points refers to a low level of resilience, a range from 67 to 72 points suggests a moderate level of resilience and 73-91 points indicate a high level of resilience.

Sociodemographic variables and medical history were collected from each participant's medical record. The following variables of interest were selected from the electronic medical record: age, gender, date of diagnosis, laboratory tests (haemoglobin, creatinine, calcium, serum-free light chains, monoclonal protein in serum and urine), presence of lytic bone lesions at the time of diagnosis and comorbidities. Comorbid conditions were used to calculate the Charlson Comorbidity Index. ${ }^{24}$ For multivariate analyses, we had to consider different categories of MM. Therefore, classification was determined according to the guidelines of the International Myeloma Working Group ${ }^{25}$ as follows: new diagnosis of MM (before treatment) or treated MM and MGUS or SMM.

\section{Statistical analysis}

Multivariate regression models were estimated to determine the impact of resilience levels (low(referent)/ average/high) on the variables of interest. We performed multiple linear regression analyses for continuous variables (MCS/PCS) and ordered logistic regression for a categorical variable (depression severity). A reference model was defined, comprised a set of the following control variables: age, gender (referent: female) and stage of myeloma (referent: MGUS or SMM). All analyses were two sided; $p$ values below 0.05 were considered statistically significant. In additional sensitivity analyses, we performed all analyses with continuous scores (of the
PHQ-9 and the RS-13, respectively) instead of categorical variables. Further, we included an interaction term (disease stage $\times$ resilience score) to investigate possible differences in the associations between resilience and the various outcomes (HRQOL and depression, respectively) for the different stages of myeloma. All statistical analyses were performed using $\mathrm{R}$ statistical software (V.3.3.2).

\section{Patient and public involvement}

Patients were not involved in the recruitment to and conduct of the study. Results will be disseminated to study participants through annual information events and contact with self-help groups.

\section{RESULTS}

\section{Study sample}

During the study period, 292 out of 578 eligible Patients (response rate: $50.5 \%$ ) were enrolled (figure 1). Mean age of the sample was 62.5 years with a range from 30.9 to 87.5 years. The majority of the patients was male $(n=173$; $59.2 \%)$. Ninety-eight patients $(33.6 \%)$ had a new diagnosis of active MM, 106 patients (36.3\%) were already treated for MM and 88 patients had the diagnosis of a precursor (MGUS or SMM; 30.1\%) of MM. High level of resilience, according to the RS-13 was present in $57.3 \%$ of the sample, $15.6 \%$ had a moderate and $27.1 \%$ reported a low level of resilience. Mean values of the SF-12 sum scores were 43.2 for MCS (range 11.5-68.9) and 37.5 for PCS (range 16.4-59.6), respectively. Moderate to severe depressive symptoms, according to the PHQ-9, were prevalent in $21 \%$ of all participants. Characteristics of the entire sample are shown in table 1.

\section{Non-responder analyses}

We analysed differences in age, sex and disease stage between study participants $(\mathrm{n}=292)$ and non-participants

\section{STROBE Flow Diagram}

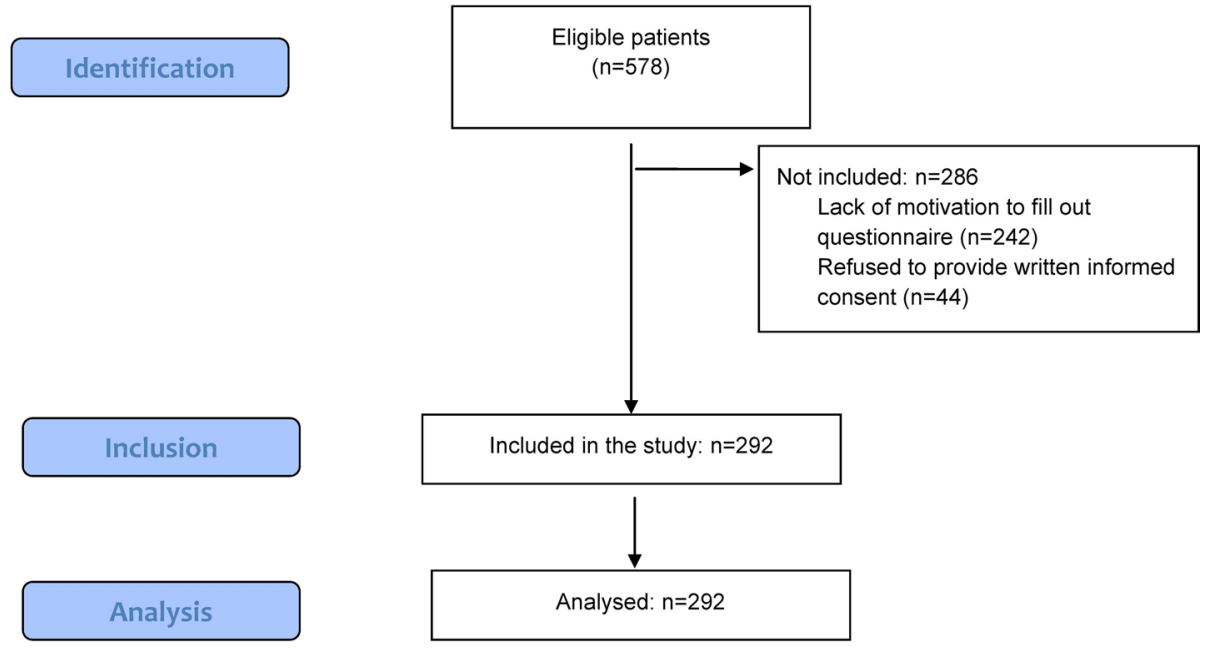

Figure 1 Study enrolment flow chart. STROBE, Strengthening the Reporting of Observational Studies in Epidemiology. 
Table 1 Characteristics of the study sample

\begin{tabular}{|c|c|c|}
\hline Variables & $\mathbf{N}$ & \\
\hline All patients & 292 & $(100 \%)$ \\
\hline Age, median (range) & & $62.5(30.9-87.5)$ \\
\hline \multicolumn{3}{|l|}{ Sex } \\
\hline Female & 119 & $(40.8 \%)$ \\
\hline Male & 173 & $(59.2 \%)$ \\
\hline \multicolumn{3}{|l|}{ Stage of MM } \\
\hline New diagnosis of MM & 98 & $(33.6 \%)$ \\
\hline Treated MM & 106 & $(36.3 \%)$ \\
\hline $\begin{array}{l}\text { Premalignant stage } \\
\text { (MGUS; SMM) }\end{array}$ & 88 & $(30.1 \%)$ \\
\hline \multicolumn{3}{|l|}{ Comorbidity } \\
\hline $\begin{array}{l}\text { Charlson Comorbidity } \\
\text { Index } \leq 2\end{array}$ & 224 & $(76.7 \%)$ \\
\hline $\begin{array}{l}\text { Charlson Comorbidity } \\
\text { Index }>2\end{array}$ & 68 & $(23.3 \%)$ \\
\hline \multicolumn{3}{|c|}{ Health-related quality of life } \\
\hline PCS, median (range) & 286 & $37.5(16.4-59.6)$ \\
\hline MCS, median (range) & 286 & $43.2(11.5-68.9)$ \\
\hline \multicolumn{3}{|c|}{$\begin{array}{l}\text { Depression severity (PHQ-9 } \\
\text { sum score) }\end{array}$} \\
\hline None (0-4) & 123 & $(45.2 \%)$ \\
\hline Mild (4-9) & 92 & $(33.8 \%)$ \\
\hline Medium (10-14) & 34 & $(12.5 \%)$ \\
\hline Severe (15-27) & 23 & $(8.5 \%)$ \\
\hline
\end{tabular}

MM, multiple myeloma; MGUS, monoclonal gammopathy of undetermined significance; PCS, Physical Component Score; PHQ-9, Patient Health Questionnaire-9; SMM, smouldering multiple myeloma.

$(\mathrm{n}=286)$. No statistically significant differences were found with regard to age (participants: median $=62.5 \mathrm{vs}$ non-participants: median $=62.9 ; \mathrm{p}=0.77$ ), sex (participants: female $\mathrm{n}=119(40.8 \%)$ vs non-participants: female $\mathrm{n}=114(39.9 \%) ; \mathrm{p}=0.89)$ and disease stage (participants: MGUS, SMM n=88 (30.1\%); new diagnosis of MM n=98 $(33.6 \%)$; treated MM n=106 (36.3\%) vs non-participants: MGUS, SMM n=95 (33.2\%); new diagnosis of MM n=73 $(25.5 \%)$; treated MM $\mathrm{n}=118(41.3 \%) ; \mathrm{p}=0.11)$

Results of multiple regression analyses for HRQOL

Table 2 and table 3 show the results of the multiple linear regression analyses for MCS and PCS. As mentioned above, our dependent (outcome) variables were MCS and PCS, respectively. A high level of resilience was significantly associated with elevated MCS $(\mathrm{p}<0.001)$. No significant association was found between a moderate level of resilience and MCS $(p=0.14)$. Further, a high level of resilience was significantly associated with PCS $(p<0.001)$. No significant association was found between a moderate level of resilience and PCS $(\mathrm{p}=0.39)$.

Results of multiple regression analysis for depression severity Table 4 shows the results of the multiple regression analysis for depression severity as dependent outcome variable. Multivariate ordered logistic regression analysis showed that the odds for higher depression severity were significantly lowered for individuals with a high level of resilience compared with the individuals with a low level of resilience (OR 0.11; $<<0.001)$. Individuals with moderate level of resilience showed a trend towards significance with lowered odds for higher depression severity (OR $0.51 ; \mathrm{p}=0.06)$.

\section{Sensitivity analyses}

Linear regression analyses with the continuous resilience variable showed a significant linear association with the respective outcomes of PCS/MCS (positive association) and continuous PHQ-9 score (negative association). Graphical inspection of the data did not indicate quadratic or other non-linear relationships. The results are shown in online supplementary tables $1-3$.

Table 2 Results of the multiple linear regression analyses with resilience as independent variable and HRQOL Physical Component Score as outcome variable adjusted by age, sex and stage of MM (premalignant stage: MGUS or SMM)

\begin{tabular}{llc}
\hline Variable & $\mathbf{b}^{*} \mathbf{( 9 5 \% \mathbf { C l } )}$ & P values \\
\hline Level of resilience; reference low level & & 0.39 \\
$\quad$ Moderate level of resilience & $1.65(-2.12$ to 5.41$)$ & $<0.001 \dagger$ \\
High level of resilience & $7.20(4.43$ to 9.98$)$ & $0.002 \dagger$ \\
Age; 5 years increase & $-0.88(-1.44$ to -0.33$)$ & 0.76 \\
Sex; reference: female & $0.37(-2.06$ to 2.80$)$ & $0.02 \dagger$ \\
Stage of MM; reference: premalignant stage & & $<0.001 \dagger$ \\
$\quad$ New diagnosis of MM & $-3.56(-6.55$ to -0.57$)$ & $-7.78(-10.65$ to -4.81$)$ \\
\hline
\end{tabular}

${ }^{*}$ Regression coefficient: the usual interpretation of a regression coefficient is the average change in the outcome variable when the corresponding predictor variable is changed by one unit.

tp-value $<0.05$.

$\ddagger$ Premalignant stage: MGUS or SMM.

HRQOL, health-related quality of life; MM, multiple myeloma; MGUS, monoclonal gammopathy of undetermined significance; SMM, smouldering multiple myeloma. 
Table 3 Results of the multiple linear regression analyses with resilience as independent variable and HRQOL (MCS) as outcome variable adjusted by age, sex and stage of MM

\begin{tabular}{lcc}
\hline Variable & $\mathbf{b}^{*}(\mathbf{9 5 \%} \mathbf{C l})$ & P values \\
\hline Level of resilience; reference low level & & 0.14 \\
$\quad$ Moderate level of resilience & $2.81(-0.92$ to 6.54$)$ & $<0.001 \dagger$ \\
$\quad$ High level of resilience & $12.12(9.36$ to 14.87$)$ & 0.3 \\
Age; 5 years increase & $0.29(-0.26$ to 0.84$)$ & $0.0012 \dagger$ \\
Sex; reference: female & $4.01(1.60$ to 6.41$)$ & 0.96 \\
Stage MM; reference: premalignant stage & & 0.53 \\
$\quad$ New diagnosis of MM & $-0.07(-3.03$ to 2.89$)$ & $-0.92(-3.81$ to 1.97$)$ \\
\hline
\end{tabular}

${ }^{*}$ Regression coefficient: the usual interpretation of a regression coefficient is the average change in the outcome variable when the corresponding predictor variable is changed by one unit.

tp-value $<0.05$.

$\ddagger$ Premalignant stage: monoclonal gammopathy of undetermined significance or smouldering multiple myeloma.

HRQOL, health-related quality of life; MCS, Mental Component Score; MM, multiple myeloma.

\section{Moderator analyses}

Moderator analyses revealed that stage of myeloma moderates the association between resilience and HRQOL and depression, respectively. The association between resilience and mental quality of life (MCS) was significantly stronger in patients with a new diagnosis. Further, the association between resilience and physical quality of life (PCS) was significantly weaker in patients with treated MM. With regard to PCS in the 'treated MM' group, the remaining effect of resilience was very small. Further, the inverse association of resilience and depression was significantly stronger in patients with a new diagnosis of MM. Results of moderator analyses are shown in online supplementary tables $1-3$.

\section{DISCUSSION}

\section{Principal findings}

In this cross-sectional study of 292 patients with monoclonal gammopathies including patients with MM as well as premalignant stages (MGUS and SMM), we found primarily that patients with a high level of resilience had a better HRQOL according to physical (PCS) and mental (PCS) sum scores of the SF-12. Furthermore, patients with a moderate or high level of resilience had significantly decreased odds of having moderate or severe depressive symptoms. Moderator analyses revealed that resilience had a stronger impact on MCS and depression in patients with a new diagnosis (before treatment). The association between resilience and physical quality of life (PCS) was significantly weaker in patients with treated MM.

\section{Comparison to other studies in the field}

Our results are in line with a smaller study of Schumacher et al that were the first who demonstrated a link between resilience and HRQOL in a rather small and heterogeneous sample of 75 haematological patients after stem cell transplantation (SCT) ${ }^{26}$ Due to the heterogeneity of the patient cohort (including patients with leukaemia, lymphoma, myeloma and aplastic anaemia) in a specific clinical situation (of SCT), a comparison to our study appears to be rather complicated.

Table 4 Results of the ordered logistic regression analysis with resilience as independent variable and depression severity (no/low/medium/severe) as outcome variable adjusted by age, sex and stage of MM

\begin{tabular}{llc}
\hline Variable & OR (95\% Cl) & P values \\
\hline Level of resilience; reference low level & & 0.06 \\
$\quad$ Moderate level of resilience & $0.51(0.24$ to 0.98$)$ & $<0.001^{*}$ \\
High level of resilience & $0.11(0.06$ to 0.19$)$ & 0.88 \\
Age: 5 years increase & $1(0.98$ to 1.03$)$ & 0.06 \\
Sex: reference female & $0.63(0.39$ to 1.02$)$ & 0.31 \\
Stage of myeloma: premalignant stage† & & $0.0046^{\star}$ \\
$\quad$ New diagnosis of MM & $1.39(0.74$ to 2.61$)$ & $2.38(1.32$ to 4.38$)$ \\
\hline Treated MM & & \\
\hline
\end{tabular}

${ }^{*} p$-value $<0.05$.

†Premalignant stage: monoclonal gammopathy of undetermined significance or smouldering multiple myeloma.

MM, multiple myeloma. 
We found a positive link between resilience and physical aspects of HRQOL. This corresponds to results from other studies that reported reduced symptom severity and side effects such as fatigue, pain or insomnia in patients with high levels of resilience. ${ }^{12}{ }^{27}$ Resilience seems to mediate the relationship between cancer symptom distress and HRQOL. ${ }^{14}$ In our sample, resilience was strongly associated to mental HRQOL and inversely related to depression symptom severity. These results are consistent with findings from studies in other cancer entities: Sharpley et al observed a negative association between resilience and depression in 425 patients with prostate cancer. ${ }^{28}$ Further studies suggest relations between high levels of resilience and lower levels of anxiety and depression. ${ }^{29}$

\section{Significance of resilience in the course of monoclonal plasma cell disorders}

The regression analyses show that the stage of the disease was significantly related to PCS but not to MCS. This appears to be plausible because physical problems increase in later phases of the disease, while mental burden seems to be high in all phases.

Moderator analyses reveal that resilience has a stronger link to MCS and depression in patients with a new diagnosis and a weaker association to PCS in patients with treated MM.

With regard to MM, one can imagine that patients at an early stage (before the start of therapy) are particularly challenged to anticipate coping with the disease and the upcoming therapy. In our sample, resilience could have a stronger protective effect on mental aspects of HRQOL and depression in early (untreated) stages, than at a later point in time, if the burden by the demanding therapy such as high-dose chemotherapy with ASCT determines the state of perceived mental health. Physical aspects of quality of life (PCS) hardly seem to be influenced by resilience in treated patients, since other factors (eg, symptoms of illness, side effects of therapy) probably play an overwhelming role. However, to confirm this conclusion, longitudinal studies must be carried out in which patients are observed over a longer period of time.

\section{Definition of resilience and its role in cancer}

In the current scientific discussion, resilience is described as a multidimensional construct that encompasses both, relatively stable personality traits and a state that is triggered by adverse events (AEs) such as undergoing a treatment for cancer. ${ }^{30}$ In a recent study of Markovitz et al, the results suggest that resilience has a stronger relation to psychosocial factors in patients with cancer compared with non-cancer patients. ${ }^{31}$ The authors conclude that resilience can be seen as a trait variable and may protect against emotional burden in patients with cancer. Wagnild and Young (the authors of the scale, which was used in our current study) define resilience as a personality trait, which has a moderating effect on negative feelings and stress and a flexible adaptation to adverse situations. ${ }^{23}$ In a recently published comprehensive review of the literature, Deshields et al give an overview of the large body of resilience literature in oncology. ${ }^{32}$ Further, the authors propose an interesting model of resilience in adult patients with cancer based on the literature and extensive clinical experience. The researchers 'take the position that resilience is both an outcome and a dynamic process that can be fostered.... ${ }^{32}$ Resilience can be seen as a common response to cancer diagnosis and treatment. According to the model by Richardson, ${ }^{33}$ personal traits and environmental circumstances as well as experiences after diagnosis/treatment of cancer may contribute to resilience which is defined as a dynamic process. In the future, longitudinal studies should clarify whether resilience is stable or changes in the course of monoclonal plasma cell disorders and other diseases.

\section{Clinical implications}

The concept of resilience is promising for its potential application in interventional research in order to support patients with MGUS, SMM and MM to cope with the situation. In a recently conducted systematic review and meta-analysis, modest effects of resiliency training were reported. ${ }^{34}$ However, only 2 of the 25 studies included focused on patients with cancer without considering haematological malignancies. ${ }^{35}{ }^{36}$ In a sample of ambulatory patients with cancer, high levels of resilience were strongly associated with lower levels of unmet supportive care needs. ${ }^{37}$ Therefore, within a complex intervention for supportive care in cancer the researchers tried to facilitate resilience and reduce unmet supportive care needs. ${ }^{38}$ Resilience levels could only be significantly influenced in a few participants. It must be concluded that it remains unclear whether resilience can be trained in patients with MM and other cancer entities.

\section{Strengths and limitations}

The major strength of this study is the large clinical sample and that most data are based on physician-recorded information. HRQOL and other psychosocial constructs were measured with validated questionnaires. A further strength of this analysis is the consideration of all stages of monoclonal plasma cell disorders including MGUS and SMM.

The main limitation of our study is related to its cross-sectional design that does not allow for either temporal or causal inferences. Further studies with consecutive measurements are needed to gain deeper knowledge of psychosocial and physical factors that could be linked to resilience in MM.

Second, in our study, we had no healthy control group to investigate differences of resilience level and relations to our outcome measures.

Third, for measurement of HRQOL, we used a generic questionnaire. Disease-specific questionnaires, such as the Core Quality of Life Questionnaire - 30 or the Multiple Myeloma Module-20, allow to quantify several disease-specific aspects of HRQOL (such as fatigue and nausea) and symptoms of myeloma, respectively. ${ }^{39}$ Previously, Jordan 
et al could show that both severity and type of disease symptoms, and treatment-related AEs are all important determinants of HRQOL in patients with MM. ${ }^{40}$ Further, disease-specific measures seem to be more sensitive for the detection of small changes in interventional or longitudinal studies. ${ }^{29}$ However, clinicians report, that even (asymptomatic) precursors of MM (that are not treated yet) seem to be associated with a deterioration of HRQOL and augmented psychological burden. Therefore, we used a generic measure that offers the opportunity to compare outcomes across different populations including asymptomatic prestages of MM. Furthermore, the SF-12 is one of the most widely used generic questionnaires in HRQOL research. In future longitudinal observations, a combination of disease-specific and generic measures is recommended to detect small changes in HRQOL. ${ }^{41}$

\section{CONCLUSION}

In conclusion, resilience may be a protective factor in the disease trajectory of MM and its precursors. As a next step, future research should focus on longitudinal assessments at various time points to elucidate the role of resilience in one of the most frequent haematological malignancies.

\section{Author affiliations}

${ }^{1}$ Department of General Internal Medicine and Psychosomatics, Medical University Hospital Heidelberg, Heidelberg, Germany

${ }^{2}$ Section of Psychooncology, National Center for Tumor Diseases, Heidelberg, Germany

${ }^{3}$ Department of Hematology, Oncology and Rheumatology, Medical University Hospital Heidelberg, Heidelberg, Germany

${ }^{4}$ Department of Biostatistics, German Cancer Research Center, Heidelberg, Germany ${ }^{5}$ Department of Orthopedics and Traumatology, University Hospital Heidelberg, Heidelberg, Germany

${ }^{6}$ Section of Multiple Myeloma, National Center for Tumor Diseases, Heidelberg, Germany

Acknowledgements The authors would like to thank the participants who made this study possible. We acknowledge financial support by Deutsche Forschungsgemeinschaftwithin the funding programme Open Access Publishing, by the Baden-WürttembergMinistry of Science, Research and the Arts and by Ruprecht-Karls-UniversitätHeidelberg.

Contributors IM: conceptualisation, data curation, formal analysis, methodology, writing original draft, and writing - review and editing. SH: investigation, data curation, methodology, project administration, visualisation, writing — review and editing. NB and MH: data curation, formal analysis, software, methodology, visualisation, writing —original draft and writing — review and editing. SH: conceptualisation, methodology, writing — review and editing. MH: project administration, conceptualisation, data curation, investigation, and writing - review and editing. HG: project administration, conceptualisation, supervision, data curation, and writing —review and editing. MH: conceptualisation, methodology, supervision, data curation, and writing — review and editing. WH: project administration, conceptualisation, supervision, data curation, investigation, and writing — review and editing. DS: data curation, formal analysis, software, methodology, writing —original draft and writing — review and editing. JH: conceptualisation, investigation, data curation, formal analysis, methodology, writing — original draft, and writing — review and editing.

Funding The authors have not declared a specific grant for this research from any funding agency in the public, commercial or not-for-profit sectors.

Competing interests None declared.

Patient consent Not required.
Ethics approval Ethical approval was obtained from the ethics committee of the Medical Faculty of Heidelberg.

Provenance and peer review Not commissioned; externally peer reviewed.

Data sharing statement Data are available (for researchers) from the corresponding author.

Open access This is an open access article distributed in accordance with the Creative Commons Attribution Non Commercial (CC BY-NC 4.0) license, which permits others to distribute, remix, adapt, build upon this work non-commercially, and license their derivative works on different terms, provided the original work is properly cited, appropriate credit is given, any changes made indicated, and the use is non-commercial. See: http:// creativecommons.org/licenses/by-nc/4.0/.

\section{REFERENCES}

1. Kyle RA, Remstein ED, Therneau TM, et al. Clinical course and prognosis of smoldering (asymptomatic) multiple myeloma. $N$ Engl J Med 2007;356:2582-90.

2. Landgren O, Kyle RA, Pfeiffer RM, et al. Monoclonal gammopathy of undetermined significance (MGUS) consistently precedes multiple myeloma: a prospective study. Blood 2009;113:5412-7.

3. Kristinsson SY, Anderson WF, Landgren O. Improved long-term survival in multiple myeloma up to the age of 80 years. Leukemia 2014;28:1346-8.

4. Kvam AK, Waage A. Health-related quality of life in patients with multiple myeloma--does it matter? Haematologica 2015;100:704-5.

5. Nielsen LK, Jarden M, Andersen CL, et al. A systematic review of health-related quality of life in longitudinal studies of myeloma patients. Eur J Haematol 2017;99:3-17.

6. Testa MA, Simonson DC. Assessment of quality-of-life outcomes. $N$ Engl J Med 1996;334:835-40.

7. Lamers J, Hartmann M, Goldschmidt H, et al. Psychosocial support in patients with multiple myeloma at time of diagnosis: who wants what? Psychooncology 2013;22:n/a-20.

8. Molassiotis A, Wilson B, Blair S, et al. Unmet supportive care needs, psychological well-being and quality of life in patients living with multiple myeloma and their partners. Psychooncology 2011;20:88-97.

9. Strasser-Weippl K, Ludwig H. Psychosocial QOL is an independent predictor of overall survival in newly diagnosed patients with multiple myeloma. Eur J Haematol 2008;81:374-9.

10. Eicher M, Matzka M, Dubey C, et al. Resilience in adult cancer care: an integrative literature review. Oncol Nurs Forum 2015;42:E3-E16.

11. Ye ZJ, Qiu HZ, Li PF, Zj Y, Pf L, et al. Predicting changes in quality of life and emotional distress in Chinese patients with lung, gastric, and colon-rectal cancer diagnoses: the role of psychological resilience. Psychooncology 2017;26:829-35

12. Ristevska-Dimitrovska G, Filov I, Rajchanovska D, et al. Resilience and Quality of Life in Breast Cancer Patients. Open Access Maced J Med Sci 2015;3:727-31.

13. Manne SL, Myers-Virtue S, Kashy D, et al. Resilience, Positive Coping, and Quality of Life Among Women Newly Diagnosed With Gynecological Cancers. Cancer Nurs 2015;38:375-82.

14. Wu WW, Tsai SY, Liang SY, et al. The Mediating Role of Resilience on Quality of Life and Cancer Symptom Distress in Adolescent Patients With Cancer. J Pediatr Oncol Nurs 2015;32:304-13.

15. Min JA, Yoon S, Lee CU, et al. Psychological resilience contributes to low emotional distress in cancer patients. Support Care Cancer 2013;21:2469-76.

16. Matzka M, Mayer H, Köck-Hódi S, et al. Relationship between Resilience, Psychological Distress and Physical Activity in Cancer Patients: A Cross-Sectional Observation Study. PLoS One 2016;11:e0154496

17. Faller H, Schuler M, Richard M, et al. Effects of psycho-oncologic interventions on emotional distress and quality of life in adult patients with cancer: systematic review and meta-analysis. J Clin Oncol 2013;31:782-93.

18. Ware J, Kosinski M, Keller SD. A 12-Item Short-Form Health Survey: construction of scales and preliminary tests of reliability and validity. Med Care 1996;34:220-33.

19. Wirtz MA, Morfeld M, Glaesmer $\mathrm{H}$, et al. Konfirmatorische Prüfung der Skalenstruktur des SF-12 Version 2.0 in einer deutschen bevölkerungsrepräsentativen Stichprobe. Diagnostica 2017.

20. Gräfe K, Zipfel S, Herzog W, et al. Screening psychischer Störungen mit dem "Gesundheitsfragebogen für Patienten (PHQ-D)“. Diagnostica 2004;50:171-81. 
21. Kroenke K, Spitzer RL, Williams JB, et al. The Patient Health Questionnaire Somatic, Anxiety, and Depressive Symptom Scales: a systematic review. Gen Hosp Psychiatry 2010;32:345-59.

22. Leppert K, Koch B, Brähler E, et al. Die Resilienzskala (RS)Überprüfung der Langform RS-25 und einer Kurzform RS-13. Klinische Diagnostik und Evaluation 2008;1:226-43.

23. Wagnild GM, Young HM. Development and psychometric evaluation of the Resilience Scale. J Nurs Meas 1993;1:165-78.

24. Charlson M, Szatrowski TP, Peterson J, et al. Validation of a combined comorbidity index. J Clin Epidemiol 1994;47:1245-51.

25. Rajkumar SV, Dimopoulos MA, Palumbo A, et al. International Myeloma Working Group updated criteria for the diagnosis of multiple myeloma. Lancet Oncol 2014;15:e538-e548.

26. Schumacher A, Sauerland C, Silling G, et al. Resilience in patients after allogeneic stem cell transplantation. Support Care Cancer 2014;22:487-93.

27. Strauss B, Brix C, Fischer S, et al. The influence of resilience on fatigue in cancer patients undergoing radiation therapy (RT). $J$ Cancer Res Clin Oncol 2007;133:511-8.

28. Sharpley CF, Bitsika V, Wootten AC, et al. Does resilience 'buffer' against depression in prostate cancer patients? A multi-site replication study. Eur J Cancer Care 2014;23:545-52.

29. Hu T, Xiao J, Peng J, et al. Relationship between resilience, social support as well as anxiety/depression of lung cancer patients: A cross-sectional observation study. J Cancer Res Ther 2018;14:72-7.

30. Aburn G, Gott M, Hoare K. What is resilience? An Integrative Review of the empirical literature. J Adv Nurs 2016;72:980-1000.

31. Markovitz SE, Schrooten W, Arntz A, et al. Resilience as a predictor for emotional response to the diagnosis and surgery in breast cancer patients. Psychooncology 2015;24:1639-45.

32. Deshields TL, Heiland MF, Kracen AC, et al. Resilience in adults with cancer: development of a conceptual model. Psychooncology 2016;25:11-18.
33. Richardson GE. The metatheory of resilience and resiliency. $J$ Clin Psychol 2002;58:307-21.

34. Leppin AL, Bora PR, Tilburt JC, et al. The efficacy of resiliency training programs: a systematic review and meta-analysis of randomized trials. PLoS One 2014;9:e111420.

35. Loprinzi CE, Prasad K, Schroeder DR, et al. Stress Management and Resilience Training (SMART) program to decrease stress and enhance resilience among breast cancer survivors: a pilot randomized clinical trial. Clin Breast Cancer 2011;11:364-8.

36. Sahler OJ, Dolgin MJ, Phipps S, et al. Specificity of problemsolving skills training in mothers of children newly diagnosed with cancer: results of a multisite randomized clinical trial. J Clin Oncol 2013;31:1329-35.

37. Dubey C, De Maria J, Hoeppli C, et al. Resilience and unmet supportive care needs in patients with cancer during early treatment: A descriptive study. Eur J Oncol Nurs 2015;19:582-8.

38. Eicher M, Ribi K, Senn-Dubey C, et al. Interprofessional, psychosocial intervention to facilitate resilience and reduce supportive care needs for patients with cancer: Results of a noncomparative, randomized phase II trial. Psychooncology 2018.

39. Cocks K, Cohen D, Wisløff F, et al. An international field study of the reliability and validity of a disease-specific questionnaire module (the QLQ-MY20) in assessing the quality of life of patients with multiple myeloma. Eur J Cancer 2007;43:1670-8.

40. Jordan K, Proskorovsky I, Lewis P, et al. Effect of general symptom level, specific adverse events, treatment patterns, and patient characteristics on health-related quality of life in patients with multiple myeloma: results of a European, multicenter cohort study. Support Care Cancer 2014;22:417-26.

41. Patrick DL, Deyo RA. Generic and disease-specific measures in assessing health status and quality of life. Med Care 1989;27(3 Suppl):S217-S232. 